\title{
Automation and User Interaction Schemes for Home Energy Management - A Combined Approach
}

\author{
David Rua, Cláudia Abreu, Tiago Costa \\ INESC TEC \\ Porto, Portugal \\ \{drua, claudia.r.abreu, tacosta\}@inesctec.pt
}

\author{
Miguel Heleno \\ Berkeley Lab - Grid Integration Group \\ Berkeley, United States \\ MiguelHeleno@lbl.gov
}

\begin{abstract}
This paper presents the development framework for an energy management platform that is being developed within the AnyPLACE project. In order to ensure that end-users become active participants in services like demand response, a combined approach is necessary in terms of monitoring, automation, and user interfacing. The success in engaging the end-user, as the centerpiece of the energy management challenge, is vital in taking advantage of a more efficient use of energy, as it is shown in this paper. The proposed framework can be run in a single board computer.
\end{abstract}

Keywords—energy optimization; user interface; monitoring; automation; self-learning techniques.

\section{INTRODUCTION}

The advent of the smart grid paradigm has been used to implement new energy services that include new stakeholders. Among the new participants are consumers, which are abandoning their passive role to become aware of their potential role in better manage their energy expenditures.

The implementation of energy services like Demand Response (DR) depends largely on the participation of consumers with whom contracts need to be established to ensure the provision of such service. Retailers and aggregators make use of the combined participation of different consumers (some are also producers) of energy to ensure the stabilization of the aggregated consumption and generation profiles and thus ensures a more confident market participation.

In the case of domestic consumers, the challenge is considerable since separately the energy profile can vary significantly from consumer to consumer but also because their engagement in services like DR is still far from being a typical situation. The implementation of DR lies on the exploitation of existing flexibility, like trading-off in terms of comfort to gain financial advantage, which is something understood by consumers but not very desirable; the temporal shift of loads is yet another strategy to ensure flexibility but it requires a schedule to be produced which is supported by manual or automatic actions.

As the existing Home Energy Management Solutions (HEMS) solutions are ineffective in producing the necessary engagement of end-user and consequently the advertised energy efficiency or savings targets, there is a need for technological platforms that allow the consumer to monitor the

This work is supported by the AnyPLACE H2020 EU project from the EU Horizon 2020 Framework Programme for Research and Innovation, under grant agreement No. 646580. energy consumption and provide them with an alternative to the typical energy usage profile.

This paper introduces a development framework to allow the successful implementation of a true energy management platform that focuses on the needs of the consumer. This platform, developed within the H2020 EU Project AnyPLACE, favors the use automated monitoring and control schemes paired with an appropriate user-interaction approach to maximize the engagement and long term participation. This platform, hereinafter designated by AnyPLACE platform, is ran in a budget single board computer as a way to keep implementation costs low.

The platform, being composed by software and hardware solutions, consists in a cluster of modules interconnected with each other and the respective interface with the end-user, devices and monitoring area Fig. 1. However, in this paper, only the modules directly related with the end user will be described.

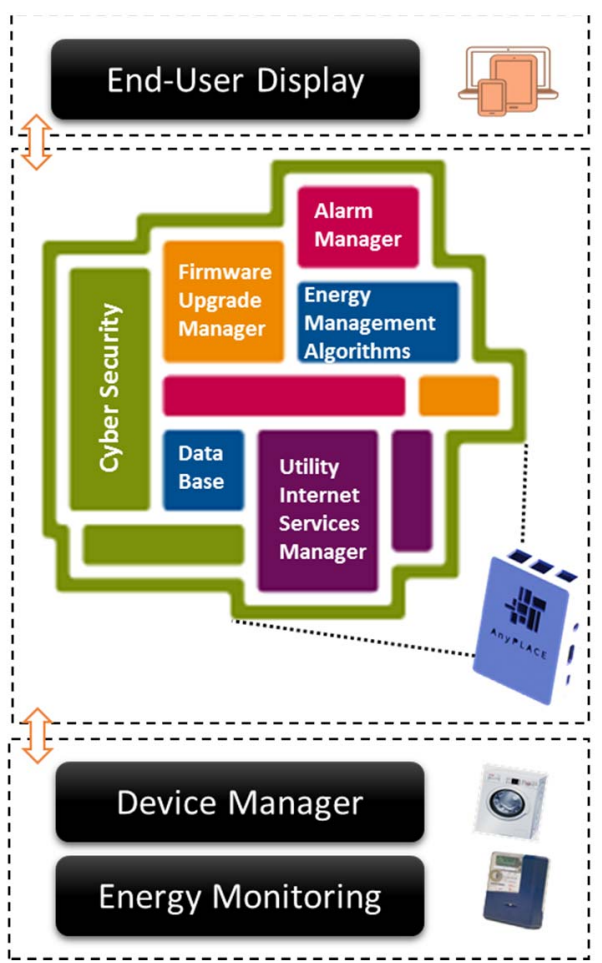

Fig. 1 AnyPLACE platform 
Being a project currently in progress, the AnyPLACE platform is undergoing various conceptual and initial development discussions. As for the full development of the platform, it is scheduled to start at the end of Q3 2016.

\section{USER INTERFACE DESIGN}

User interface design for home automation systems has come a long way from primitive designs presented on earlier iterations of such systems. The advent of mobile computing, with devices such as smartphones or tablets, has given users the access to rich user centric interfaces, capable of showing several types of information in real time. However, simplicity has to be the main guideline for any graphical user interface (GUI) design, so that user's aren't overloaded with unnecessary data or requests on such small screens. Besides this, the user interface layer should be separated from underlying data and communication layers, used to interact with other integrated modules on the system. Previous works on this subject have shown concerns that should be present on GUI design: in [1], energy efficiency of GUI on mobile devices is explored and several techniques used to improve both battery consumption and user performance are presented. Mobile GUI design for challenging environments is the main topic in [2]. Specific restraints, such as limited screen sizes or outdoor usage are used as design guidelines for the user interface. Modularity is also addressed in this work, relying on a modified Model-viewcontroller (MVC) software pattern for layer separation. [3] presents a context-sensitive user interface, capable of showing personalized information, based on the user preferences. The GUI can also adapt to the working environment, based on context information where it is being used.

As such a GUI design had to be adaptable to the following requirements: usage on different screen sizes, ability to control all parameters of the solution and dynamic presentation of relevant data. Since the AnyPLACE platform will rely on single board computer (ex: Raspberry Pi) with integrated LCD screens and mobile applications for iOS and Android smartphones, the unified GUI had to be dynamically adaptable for both scenarios, regardless of the screen size used on any of these situations. To accomplish this feat, a "dashboard" approach was used for the AnyPLACE platform, where the total area of each screen is divided into various interactive blocks, grouped by its relevance or area. With this approach, no screen region is wasted and all information is presented to the users, using a modern and responsive user centric design. Productivity is also a major factor when designing GUI: important features should be present or accessible within reach, without any particular effort. Therefore, the AnyPLACE user interface structure was divided into several sub-screens, each one with its own responsibility:

- Main Screen: graphical presentation of historic consumption data, system overview and alarm status

- User Preferences Screen: theme selection, hardware configuration, system settings, consumption profile and alarm configurations
- Energy Management Screen: flexible loads scheduler, demand response and microgeneration control activation and tariff selection

- Device Manager Screen: appliance installation and configuration and detailed presentation of historic consumption data

- Help Screen: relevant troubleshooting options for any given module of the platform

With this structure, all relevant options of the AnyPLACE platform are available within a maximum of three touches on the screen. For example, comfort parameters such as maximum or minimum water temperature or working range of a given appliance can easily be defined by the user through the GUI. This data will then be used by the energy optimization algorithms to determine the most appropriate control strategy for the available appliances on the household, within the restrictions and preferences set by the user. Energetic usage of the GUI is also optimized, since navigational efforts from the users are reduced, with subsequent effects in the onscreen presentation of the GUI, on any particular device. Storage of GUI information (such as login and password, user preferences and local settings) is handled by a local Database Management System (DBMS) and any communication with underlying modules or devices is handled by a specific middleware developed for the AnyPLACE platform. An example of the Graphical User Interface designed for the Energy Management in the AnyPLACE platform is depicted in Fig. 2.

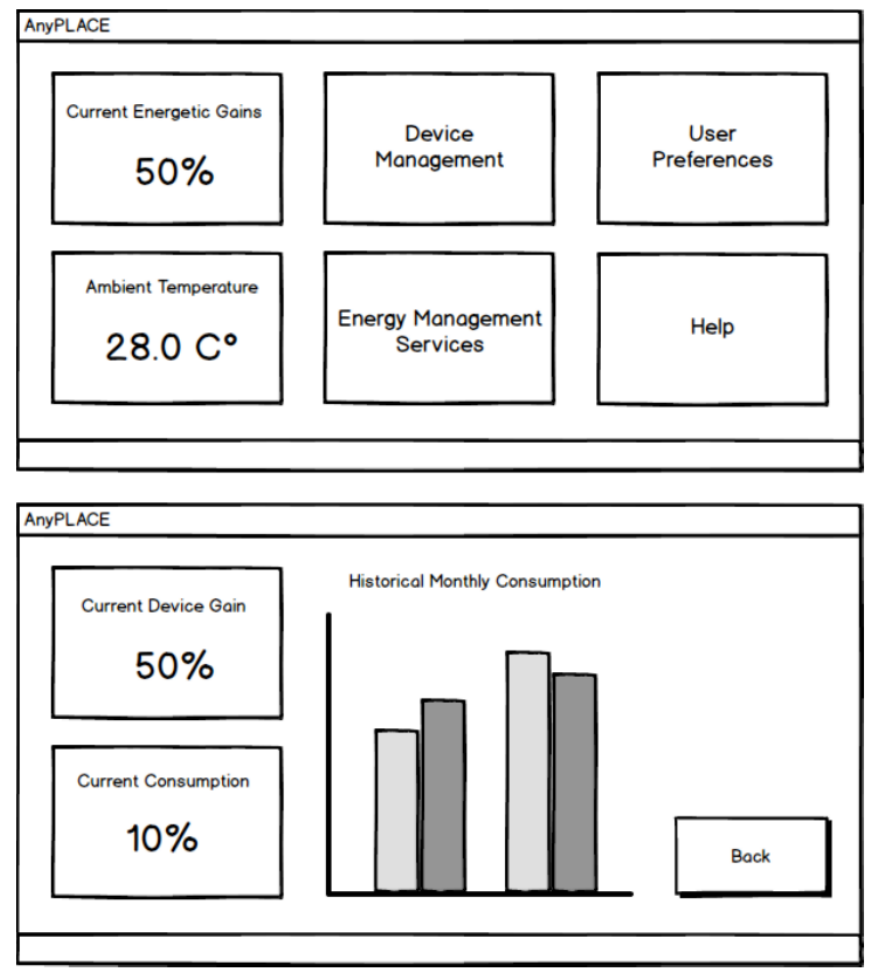

Fig. 2 Example of GUI design for the AnyPLACE solution 


\section{Automation SchEMES}

\section{A. Energy Monitoring}

A desirable characteristic for a HEMS is the ability to collect the most information possible from the surrounding context,

preferably in an automated way, to allow the energy management algorithms to produce the best (optimum) decisions. This monitoring feature can be realized with different levels of detail: aggregate through the data collection of meters (ex: electricity, gas and water); per circuit inside the building; or per plug (ex: smart plugs).

The use of different metering devices, especially those installed inside buildings, represents not only an added cost but it is also an invasive approach to maximize the information collection, often undesired. In that sense the exploitation of non-intrusive monitoring strategies is sought to enhance the data collection. These strategies rely on machine learning techniques to produce the disaggregation of load metering data, which can be used to identify the power consumption of specific appliances. This discriminated information can be used to feed-in the energy management algorithms mentioned in the section IV.

Among the different algorithms available a modified version of the Parson's algorithm is being developed [4]. This is part of a self-learning mechanism that uses aggregated electricity metering only, to provide an estimate on the discriminated use of flexible appliances (ex: thermal appliances). The objective is to focus on the use information of appliances that will be used in the energy management algorithms instead of fully disaggregating the building energy consumption. This will avoid unnecessary training sequences, which may be time consuming and computationally burden for the AnyPLACE platform.

Automated monitoring can also provide the end-user, through the appropriate user interfaces, the necessary information to allow an increased awareness of the expenditures associated to the building. This information alone contributes towards the potential increase in energy use efficiency, leveraged in different control strategies.

\section{B. Control Strategies}

Another desirable and complementary characteristic of a HEMS is the ability to schedule the operation of existing appliances (and microgeneration, if available), which can be programmed manually or automatically. The scheduling of appliances is one of the outputs of the energy management algorithms and its implementation needs to take into consideration the type of appliance or system to be controlled.

Ideally the remote control of appliance and systems should be performed in a seamless way, with the informed consent of the end-user, but without the explicit need for their activation. The main challenge is related to the fact that current appliances are not prepared to be remotely configured by a central platform like AnyPLACE. Although some appliances do allow a remote operation via local communications or via a cloud service, most of them still relies on user interaction to schedule their operation.
An alternative being explored is the use of remote plugs (also known as smart plugs) that allow the activation of appliances, but limiting the control states to either on or off. Furthermore, not all appliances will be able to be controlled this way since they may require a manual activation (ex: rearming after disconnection) or because a prolonged disconnection may lead to an undesirable state of operation (ex. restart).

The energy management algorithms need to account for all of these restrictions in terms of monitoring and control to produce optimized and realistic scheduling results.

\section{ENERGy MANAGEMENT AlgORITHMS}

The first measures to incentive efficiency in the consumption and to promote waste reduction were implemented by the electric utilities in the 80's. The first phase of DSM was born under the form of energy conservation [9]. Later, Boshell and Veloza [10] defined DSM as a relation of three terms: Energy Efficiency, Demand Response and Energy Conservation. However, the first definition of Demand Side Management was presented by Gellings [11]. According to the author, "Demand side management is the planning, implementation, and monitoring of those utility activities designed to influence customer use of electricity in ways that will produce desired changes in the utility's load shape, i.e., changes in the time pattern and magnitude of a utility's load".

Demand Side Management techniques only related to residential consumers usually aim at one or both of the following design objectives - reducing consumption and shifting consumption. This work intends to achieve both objectives. Providing a set of metering services, it allows endusers consumption awareness towards conservations and savings (behavioural approach), and by creating a variety of functionalities regarding control and management of home energy it allows the possibility of an automatic control of domestic appliances (technical approach).

The goal of the technical approach is to allow energy and cost savings due to the flexibility provided to support the electricity system operation, taking advantage of the control of microgeneration, appliances, and energy storage units and make use of smart plugs to control components that are no interoperable.

A set of algorithms were developed, to be integrated in the AnyPLACE platform, with the capability of performing a day ahead optimal scheduling of devices. This HEMS will analyse, collect data and compare costs, providing an energy awareness analysis, from an active monitoring and control of appliances.

The most complex and relevant part of these algorithms is related to the optimal scheduling of flexible devices, which is based on the work already developed in [5] for the case of thermal appliances. For AnyPLACE platform a solution was specifically created for the home domain using mixed integer programming techniques to optimize the energy use. Using consumption patterns, through the automated detection schemes of section III, as physical characteristics for all the appliances and the user preferences, the HEMS will be able to 
make an optimal scheduling for all the connected appliances that can be classified as thermal and shiftable, regarding the dynamic prices.

These appliances can be classified as shiftable and thermal, and their models are respectively based in historical data and the physically-based models. The physically-based load models are used to characterize the behaviour of residential thermostatically controlled loads [5].

Shiftable appliances operate on a predefined cycle with a known duration and consumption. The idea is to shift their operation to the low price periods. The configuration considers a deadline that is defined by the end-user as far ahead as possible with a maximum of $24 \mathrm{~h}$. A time window is available between a configuration time and deadline, during which the control method can freely choose the optimal start time.

The optimized consumption of thermal appliances can be obtained through an external on/off control schedule performed by the HEMS. The actual control temperatures are no longer the thermostat dead-band as in HEMS baseline. Instead, it is asked to the end-user to choose in the HEMS GUI the admissible comfort limits, within which the temperature should be kept by the HEMS control.

An example of an optimization performed by the AnyPLACE platform, is presented in the objective function (1), where the aim is to find the minor daily cost for the energy use. It is one of the possible criteria that can be optimized in a household. It is also constrained by various factors, as the appliances physical characteristics and end-user preferences.

$$
\begin{aligned}
& \min \left(\sum_{t=0}^{n s t e p s} C_{t} *\left[P_{t}^{s}+P_{t}^{n s}\right]\right) \\
& P_{t}^{s}=\sum_{j=1}^{n} \sum_{i=0}^{d_{j}}\left[P_{i}^{j} \times \lambda_{t-i}^{j}\right] \\
& P_{t}^{n s}=\sum_{k=1}^{m}\left[P^{m} \times P_{M C t}^{m}\right]
\end{aligned}
$$

Where,

nsteps, number of control periods in a $15 \mathrm{~min}$ interval

$\boldsymbol{n}$, number of shiftable appliances

$\boldsymbol{d}_{\boldsymbol{j}}$, duration of operation of appliance $j$

$\boldsymbol{P}_{\boldsymbol{i} \boldsymbol{j}}$, Power of period $i$ of appliance $j$

$\lambda_{t-i}^{j}$, binary variable-condition if appliance starts in period $t-i$

$\boldsymbol{P}_{\boldsymbol{M C}}^{\boldsymbol{m}}$, binary variable-condition if appliance is operating or not $\boldsymbol{m}$, number of thermal appliances

$\boldsymbol{P}^{\boldsymbol{m}}$,Capacity of thermal appliance $m$ (in $k W$ )

To create an example scenario to test the algorithm, the Spanish dynamic tariff [6] was used, along with public data sets [7] to model shiftable appliances, and standard values to model thermal appliances. The previous formulation was implemented in Java language and the GLPK solver [8] was used to run the optimization problem. This specific problem has 576 integer variables and 579 constraints (linear) and an optimal solution was produced in approximately $1 \mathrm{~h}$ for a gap of $5.0 \%$, in a Raspberry Pi 2 . The result of this simulation was the cost optimized scheduling of appliances.

The example output, presented in Fig. 3, represents the difference between the normal work (baseline) and an optimized work (modified) in a household during a 24-hour interval. The normal operating conditions (baseline scenario) of the appliances is represented by dashed columns and it shows the result of a daily operation of the appliances (their activation) in a so called typical day. The solid columns represent the modified appliance activation, as a result of the optimization algorithm described earlier, which determined the best operating time when the price curve is considered. In this new scenario no restriction is violated and as such the appliances operation is not compromised.

The thermal appliance considered in this simulation was an Air Conditioner (AC) unit, represented by the blue columns, and in Fig. 3 it is possible to verify the different hours when this appliance is activated (for both the base and new scenario). The shiftable appliance was a dishwasher (DW) (red columns), and independently of the scenario, it only works once per day as it is to be expected.

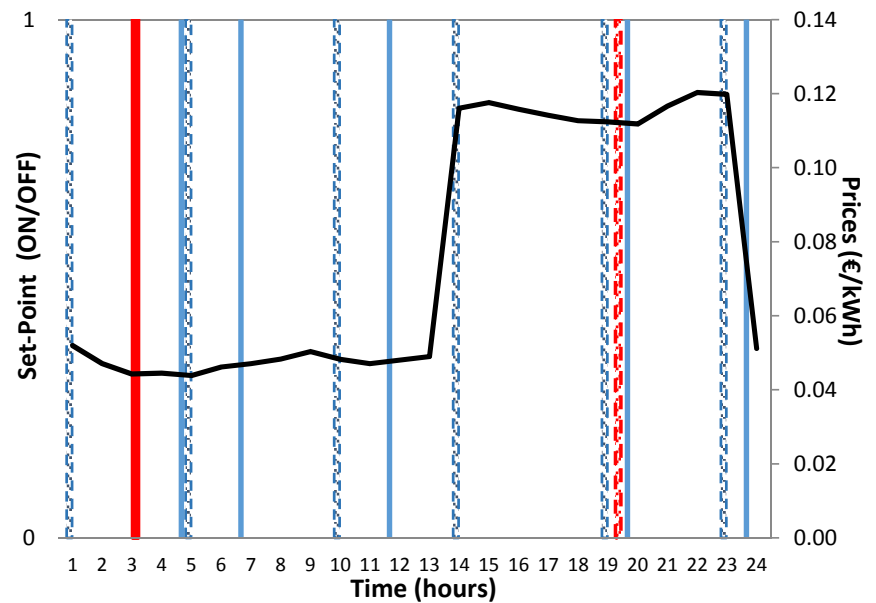

Time (hours)

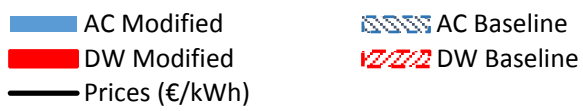

Fig. 3 Baseline and optimized profile of a thermal and a shiftable appliance regarding dynamic tariffs

Once this information is available, and the end-user is aware of the existing opportunities, an output is produced in the form of control set-points that can be sent to load devices in an automated fashion or the end-user can manually activate the appliances by following the schedule provided by the energy management algorithm. 


\section{CONSIDERATIONS}

The successful deployment of energy management schemes depends on the engagement of end-users and their active participation in redefining the way energy is used. This has not only advantages to end-users themselves but also to other stakeholders like retailers or aggregators, up to system operators that may use services like demand response to share the benefits through the different participants.

As mentioned throughout this paper a need for a combined approach is clear. The design of end-user interfaces needs to explore mechanisms to continuously incentive users to be part of the energy management, as a natural action. Automated schemes should be used as a way to maximize the extraction of data in the building context, where machine learning techniques minimize the necessary interaction with devices and systems. Energy management algorithms can make use of enhanced user interfaces and automation schemes to optimize the use of energy.

An important aspect to the implementation of a platform like the one being developed in AnyPLACE project, is the cost. The implementations described in this paper are capable of being run in a single computer board.

\section{REFERENCES}

[1] K. S. Vallerio, L. Zhong, and N. K. Jha, "Energy-Efficient Graphical User Interface Design,” IEEE Transactions on Mobile Computing, vol. 5, no. 7, pp. 846-859, July 2006

[2] R. Luostarinen, J. Manner, J. Maatta, R. Jarvinen, "User-centered design of graphical user interfaces," Military Communications Conference MILCON, pp. 50-55, August 2010

[3] J. V. Bergh, K. Coninx, "Towards Integrated Design of ContextSensitive Interactive Systems," Proceedings of the $3^{\text {rd }}$ International Conference on Pervasive Computing and Communications Workshops, pp-30-34, March 2005.

[4] O. Parson, S. Ghosh, M. Weal, A. Rogers. An Unsupervised Training Method for Nonintrusive Appliance Load Monitoring, in Artificial Intelligence. Springer 2014.

[5] M. L. D. Heleno, Phd Thesis, "Provision of Advanced Ancillary Services Through Demand Side Integration." [Online]. Available: https://repositorio-aberto.up.pt/bitstream/10216/82179/2/130410.pdf.

[6] Precio voluntario para el pequeño consumidor (PVPC) [Online]. Available: https://www.esios.ree.es/es/pvpc.

[7] O. Parson, "Disaggregated Homes," [Online]. Available: http://blog.oliverparson.co.uk/2012/06/public-data-sets-for-nialm.html.

[8] GLPK (GNU linear programming kit), 2006.

[9] Sioshansi, Fereidoom P., "Demand Side management: the third wave", Energy Policy, vol. 23, no 2. pp. 111-114, 1995.

[10] Boshell, F., Veloza, O.P., "Review of Developed Demand Side Management Programs Including Different Concepts and their Results", IEEE/PES Transmission and Distribution Conference and Exposition: Latin America, 2008.

[11] Gellings, C.W., "The concept of demand side management for electric utilities," Proceedings of the IEEE , vol.73, no.10, pp. 1468- 1470, Oct. 1985. 